\title{
Association Between Circulating Adiponectin and Heart Rate Recovery in Women With Polycystic Ovarian Syndrome
}

\author{
Huan Zheng \\ Worldpath Clinic International \\ Zhiling Li \\ Shanghai Children's Hospital,Shanghai Jiaotong University \\ Wei Feng \\ Renji Hospital, Shanghai Jiaotong University \\ Bin Liu ( $\square$ youranpachi@163.com) \\ Minhang Hospital
}

\section{Research}

Keywords: Adiponectin, Heart rate recovery, Polycystic ovary syndrome, Autonomic nervous system

Posted Date: January 22nd, 2021

DOI: https://doi.org/10.21203/rs.3.rs-151941/v1

License: (c) (i) This work is licensed under a Creative Commons Attribution 4.0 International License. Read Full License 


\section{Abstract}

Background: Imbalance of autonomic nervous system is confirmed as a key contributor for cardiometabolic complications in polycystic ovarian syndrome (PCOS) women. Heart rate recovery (HRR) is an easy test for autonomic nervous tone evaluation and a powerful index for predicting cardiovascular events and mortality. Adiponectin (APN) is reported to be correlated closely with autonomic nervous function in different populations.

Methods: This study aimed to investigate the relationship between serum total APN and HRR in PCOS women. A total of 89 PCOS women were enrolled and divided into two groups. Women with HRR values slower than 12 beats were defined as Blunted HRR Group. APN levels were compared between Blunted HRR Group and Normal HRR Group. Multivariate logistic regression analysis and multiple linear regression analysis were performed to determine which clinical variables were independently associated with decreased HRR and the effect of clinical variables on APNlevels, respectively.

Results: 23 women were categorized in Blunted HRR Group, in which the APN level was significantly lower than Normal HRR Group $(10.2 \pm 3.9 \mathrm{ug} / \mathrm{ml}$ vs. $13.1 \pm 4.1 \mathrm{ug} / \mathrm{ml}, P=0.015)$. Age [odds ratio (OR) $=1.15 ; 95 \%$ confidence interval $(\mathrm{Cl})=1.04-1.21 ; P=0.032 \mathrm{~J}$, $\mathrm{BMI}(\mathrm{OR}=0.93 ; 95 \% \mathrm{Cl}=0.72-1.08 ; P=0.006)$, hypertension $(\mathrm{OR}=1.08 ; 95 \% \mathrm{Cl}=$ 1.02-1.26; $P=0.025)$ and $\mathrm{APN}(\mathrm{OR}=0.61 ; 95 \% \mathrm{Cl}=0.43-0.88 ; P=0.018)$ were independent factors of attenuated $\mathrm{HRR}$ in PCOS women. Meanwhile, multiple linear regression analysis showed only age $(\beta=-0.26 ; 95 \% \mathrm{Cl}=-0.47--0.03, P=$ $0.041)$ and hyperlipemia $(\beta=-0.13 ; 95 \% \mathrm{Cl}=-0.27-0.08, P=0.024)$ were closely associated with APN levels in PCOS women.

Conclusions: Our findings suggested that decreased APN concentration was closely associated with HRR blunt in PCOS women. Further studies are needed to explore the underlying interactions between APN and autonomic nervous function.

\section{Background}

Polycystic ovary syndrome (PCOS) is one of the most common endocrine disorders of reproductive age, affecting $15-20 \%$ of women [1]. The 'Rotterdam' diagnostic criteria defines that women must present with two of the following three symptoms/signs - hyperandrogenism, chronic anovulation/oligomenorrhea and polycystic ovaries [2]. Epidemiological literature shows that women with PCOS have an increased prevalence of cardiometabolic complications, such as hyperlipemia, hypertension, type 2 diabetes and metabolic syndrome [3]. Although the exact etiology of PCOS has not been elucidated yet, imbalance of autonomic nervous system is confirmed as a key contributor for reproductive disorders and cardiometabolic outcomes by accumulating clinical researches [4]. Evidences for overactive function of sympathetic nervous system includes over-activation of ovarian sympathetic nerve, alternations of sympathetic neurotransmitter and increased sympathetic nerve activity of muscle vascular bed [5]. Heart rate recovery (HRR), defined as the difference between heart rate (HR) at peak exercise and HR one minute after exercise, reflects the dynamic balance and coordinated interplay between parasympathetic reactivation and sympathetic withdrawal [6]. Meta-analysis has proved that slow HRR has the prognostic value of predicting cardiovascular events and mortality in different populations, including asymptomatic adults, patients with coronary artery diseases and patients with heart failure [6]. The significance of HRR, a non-invasive and readily obtainable indicator, was verified repeatedly in those investigations, thus we hypothesized that an evaluation of HRR in PCOS women would be of clinical interest, and it would be meaningful to explore the factors which have close associations with HRR, such as adipokines. 
Adiponectin (APN), a $30 \mathrm{kDa}$ circulating serum adipokine, is predominantly secreted by adipose tissue and is downregulated in states of obesity and insulin resistance [7]. Previous studies revealed that it served as an insulinsensitizing, anti-inflammatory and vasculoprotective cytokine [8]. A recent meta-analysis by Li et al. [9], which included 1,944 women with PCOS and 1,654 healthy controls from 38 studies, reported lower APN levels in PCOS groups compared with controls. Moreover, a growing number of investigations indicated that APN was related to autonomic nervous function in adults and children [10-12]. Recently, an observation carried out in Australia reported that APN was inversely associated with sympathetic activity in PCOS women [13]. However, there were fewer similar studies focusing on the association between APN and autonomic nervous imbalance in Chinese PCOS women. In this respect, we designed this study to determine whether APN level was independently correlated with HRR in PCOS women, which would be helpful to explore the interactions between adipokines and autonomic nervous activities in PCOS.

\section{Subjects And Methods}

\section{Subjects}

This study was approved by Minhang Hospital Affiliated to Fudan University research ethics committee. Written consent was obtained from each participant before information was collected. During the period from January 2016 to November 2019, 89 women who were diagnosed as PCOS according to the Rotterdam ESHRE-ASRM-sponsored PCOS criteria [2] from the Department of Gynecology and Obstetrics were enrolled in this study. Participants were excluded if they had any of the following conditions: pregnancy, malignancy, liver or renal failure, severe orthopedic problems or severe muscular/skeletal disorders that would prohibit exercise test, acute or chronic infectious diseases, taking chronotropic drugs (i.e., ß-blockers, amiodarone, or calcium-channel-blockers) or sympathomimetic drugs within 12 weeks.

\section{Clinical data collection}

Clinical data including medical history, exercise habit (taking more than 3 times/week and 30 minutes/time regular exercise was defined as having exercise habit), smoking habit and alcohol habit were collected from each participant. Body mass index (BMI) was calculated as the ratio of weight divided by height squared $\left(\mathrm{kg} / \mathrm{m}^{2}\right)$. Systolic blood pressure $\left(\mathrm{SBP}_{\text {rest }}\right)$ at rest, diastolic blood pressure $\left(\mathrm{DBP}_{\text {rest }}\right)$ at rest and $\mathrm{HR}$ at rest $\left(\mathrm{HR}_{\text {rest }}\right)$ were also recorded.

\section{Laboratory measurement}

Blood samples were collected from each patient after at least 8 fasting hours. Fasting blood glucose(FBG), insulin(IN), aspartate aminotransferase (AST), alanine aminotransferase (ALT), total cholesterol(TC), high-density lipoprotein cholesterol(HDL-C), low-density lipoprotein cholesterol(LDL-C), triglyceride(TG) and creatinine (Cr) were measured using standard laboratory methods. Serum adiponectin level was tested by an enzyme-linked immunosorbent assay (Cyagen Bioscience Inc., Guangzhou, China), according to the manufacturer's method. The APN ELISA Kit (human) was a total APN kit detecting the sum of values of low-, middle- and high-molecular-weight APN. The intra- and inter assay coefficients of variation were $5.1 \%$ and $7.6 \%$, respectively.

\section{HRR measurement}


Every participant underwent an incremental cardiopulmonary exercise test (CPET) on a treadmill. CPET was performed according to a symptom-limited Bruce's protocol, with continuous electrocardiographic monitoring. Blood pressure (BP) was measured and recorded regularly. The test was terminated for any of the following reasons: the exertion sore greater than 17 (Borg scale); actual HR more than 90\% of age predicted maximum HR; SBP more than $200 \mathrm{mmHg}$; typical chest discomfort; severe arrhythmias; more than $1 \mathrm{~mm}$ of horizontal or downsloping ST segment depression. After achieving peak exercise, the test was almost immediately stopped. Therefore, the subjects in our CPET did not have a 'cool-down' period. HRR was obtained by subtracting HR at the first minute of recovery from peak HR obtained during exercise. It was reported that the value of HRR may be affected by the small workload during the 'cool-down', decreasing its diagnostic sensitivity $[14,15]$. The whole process was conducted by a cardiologist and a physician.

Substantial population based cohort studies verified that attenuated HRR was an independent predictor for allcause and cardiovascular mortality, although adopting different cutoff points of HRR. The value of less than 12 beats was widely used as the cutoff in those investigations, including patients referred for exercise test [16], candidates for angiography [17], survivors of acute myocardial infarction [18] and heart failure patients [19]. Therefore, we defined women whose HRR values were slower than 12 beats in our study as Blunted HRR Group. Then 89 PCOS women was divided into two subgroups: Blunted HRR Group ( $n=23)$ and Normal HRR Group ( $n=66)$.

\section{Statistical analysis}

Descriptive statistics were presented as the mean \pm SD for continuous variables or percentage for categorical variables. Differences between two groups were assessed with two-sided Fisher exact tests, chi-square tests for categorical variables and Student t-tests for continuous variables. Multivariate logistic regression analysis was performed to determine which clinical variables were independently associated with blunted HRR and the results were reported as odds ratios (ORs) and 95\% confidence intervals ( $95 \% \mathrm{Cls})$. Meanwhile, multiple linear regression analysis was used to examine the effect of clinical variables on adiponectin levels and the results were reported as $\beta$ coefficients and $95 \% \mathrm{Cls}$. All statistical analyses were performed using the software package SPSS, version 17.0 (SPSS Inc., Chicago, USA). $P<0.05$ was considered significant statistically.

\section{Results}

The demographic and clinical characteristics of 89 PCOS women were presented in Table 1. 23 patients were categorized in Blunted HRR Group. There was no women having the habit of smoking or drinking alcohol in either group. Age, BMI, PCOS duration and the prevalence of hypertension history were greater in Blunted HRR Group than those of Normal HRR Group $(P<0.05)$, while the ratio of women having exercise habit was lower in Blunted HRR Group than that of Normal HRR $\operatorname{Group}(P<0.01)$. As for $\mathrm{SBP}_{\text {rest, }} \mathrm{DBP}_{\text {rest }}, \mathrm{HR}_{\text {rest, }}$, prevalence of coronary artery disease, prevalence of hyperlipemia and prevalence of glucose metabolism disorder, there were no significant differences in the 2 groups $(P>0.05)$. In regard to biochemical indexes, there were no significant difference in FBG, Ln IN(logarithmic transformation of insulin), AST, ALT, TC, HDL-C, LDL-C, TG, and $\operatorname{Cr}(P>0.05)$. However, the APN level in Blunted HRR Group was significantly lower than that of Normal HRR Group $(P<0.05)$. 
Table 1

Comparions of clinical characteristics and laboratorymeasurements of PCOS women in 2 groups.

\begin{tabular}{|c|c|c|c|}
\hline Variables & Blunted HRR Group( $n=23)$ & Normal HRR Group $(n=66)$ & $P$-value \\
\hline Age(years) & $41.1 \pm 6.5$ & $33.7 \pm 5.2$ & 0.023 \\
\hline $\mathrm{BMI}\left(\mathrm{kg} / \mathrm{m}^{2}\right)$ & $25.4 \pm 3.3$ & $22.9 \pm 2.8$ & 0.010 \\
\hline PCOS duration(months) & $216.7 \pm 20.9$ & $148.2 \pm 24.6$ & 0.034 \\
\hline Regular exercise habit $(n, \%)$ & $5(21.7 \%)$ & $36(54.5 \%)$ & 0.007 \\
\hline $\mathrm{SBP}_{\text {rest }}(\mathrm{mmHg})$ & $127.3 \pm 15.0$ & $122.4 \pm 12.8$ & 0.126 \\
\hline $\mathrm{DBP}_{\text {rest }}(\mathrm{mmHg})$ & $70.9 \pm 11.6$ & $73.1 \pm 8.4$ & 0.248 \\
\hline $\mathrm{HR}_{\text {rest }}($ beats/min) & $76.2 \pm 9.1$ & $73.5 \pm 10.7$ & 0.516 \\
\hline Hypertension (n, \%) & $5(21.7 \%)$ & $6(9.1 \%)$ & 0.002 \\
\hline Coronary artery disease $(n, \%)$ & $0(0)$ & $0(0)$ & I \\
\hline Hyperlipemia (n, \%) & $17(73.9 \%)$ & $46(69.7 \%)$ & 0.713 \\
\hline Glucose metabolism disorder (n, \%) & $9(39.1 \%)$ & $21(32.0 \%)$ & 0.121 \\
\hline Anti-hypertension treatment $(n, \%)$ & $4(17.4 \%)$ & $4(6.1 \%)$ & $<0.001$ \\
\hline Anti-thrombotic treatment $(\mathrm{n}, \%)$ & $8(34.8 \%)$ & $4(6.1 \%)$ & 0.005 \\
\hline Lipid-lowering treatment (n, \%) & $10(43.5 \%)$ & $13(19.7 \%)$ & 0.012 \\
\hline Glucose-lowering treatment $(\mathrm{n}, \%)$ & $6(26.1 \%)$ & $15(22.7 \%)$ & 0.527 \\
\hline $\mathrm{FBG}(\mathrm{mmol} / \mathrm{L})$ & $6.1 \pm 2.3$ & $5.7 \pm 2.1$ & 0.083 \\
\hline Ln IN(mU/L) & $2.0 \pm 0.4$ & $2.1 \pm 0.4$ & 0.835 \\
\hline AST(U/L) & $31.6 \pm 5.7$ & $32.8 \pm 6.3$ & 0.419 \\
\hline $\operatorname{ALT}(\mathrm{U} / \mathrm{L})$ & $28.9 \pm 7.2$ & $30.1 \pm 5.8$ & 0.561 \\
\hline TC(mmol/L) & $5.9 \pm 1.1$ & $6.1 \pm 1.3$ & 0.274 \\
\hline $\mathrm{HDL}-\mathrm{C}(\mathrm{mmol} / \mathrm{L})$ & $0.9 \pm 0.2$ & $0.8 \pm 0.3$ & 0.243 \\
\hline LDL-C(mmol/L) & $3.7 \pm 0.9$ & $3.8 \pm 1.0$ & 0.166 \\
\hline TG(mmol/L) & $2.5 \pm 0.3$ & $2.4 \pm 0.5$ & 0.718 \\
\hline $\operatorname{Cr}(\mu \mathrm{mol} / \mathrm{L})$ & $70.5 \pm 12.6$ & $72.6 \pm 13.1$ & 0.626 \\
\hline APN(ug/ml) & $10.2 \pm 3.9$ & $13.1 \pm 4.1$ & 0.015 \\
\hline \multicolumn{4}{|c|}{$\begin{array}{l}\text { BMI, body mass index; PCOS, polycystic ovarian syndrome; SBP, systolic blood pressure; DBP, diastolic blood } \\
\text { pressure; } \mathrm{HR}_{\text {rest }} \text {, heart rate at rest; } \mathrm{FBG} \text {, fasting blood glucose; LnIN, logarithmic transformation of insulin; } A S T \text {, } \\
\text { aspartate aminotransferase; ALT, alanine aminotransferase; } \mathrm{TC} \text {, total cholesterol; } \mathrm{HDL}-\mathrm{C} \text {, high density lipoprotein } \\
\text { cholesterol; } \mathrm{LDL}-\mathrm{C} \text {, low density lipoprotein cholesterol; TG, triglyceride; } \mathrm{Cr} \text {, creatine; APN, adiponectin. }\end{array}$} \\
\hline
\end{tabular}


Multivariate logistic regression analysis identified age $(\mathrm{OR}=1.15, P<0.05), \mathrm{BMI}(\mathrm{OR}=0.93, P<0.01)$, hypertension $(\mathrm{OR}=1.08, P<0.05)$, and APN $(\mathrm{OR}=0.61, P<0.05)$ as independent variables significantly associated with blunted HRR (Table 2). The adjusted $R^{2}$ value was 0.31 for this model.

Table 2

Logistic regression analysis for blunted HRR in PCOS women.

\begin{tabular}{|lllc|}
\hline Variables & Odds ratio & $95 \% \mathrm{Cl}$ & $P$-value \\
\hline Age & 1.15 & $1.04-1.21$ & 0.032 \\
\hline BMI & 0.93 & $0.72-1.08$ & 0.006 \\
\hline Exercise habit & 0.81 & $0.70-1.12$ & 0.094 \\
\hline Hypertension & 1.08 & $1.02-1.26$ & 0.025 \\
\hline Hyperlipemia & 1.18 & $0.64-2.09$ & 0.387 \\
\hline Glucose metabolism disorder & 0.86 & $0.71-1.14$ & 0.106 \\
\hline FBG & 0.72 & $0.31-1.95$ & 0.761 \\
\hline TC & 1.24 & $1.11-1.63$ & 0.523 \\
\hline APN & 0.61 & $0.43-0.88$ & 0.018 \\
\hline BMI, body mass index; FBG, fasting blood glucose; TC, total cholesterol; APN, adiponectin. \\
\hline
\end{tabular}

Moreover, those PCOS women with hypertension had lower APN levels than those without hypertension $(P<0.05$ Table 3). There were also statistically significant differences in APN levels in both hyperlipemia and glucose metabolism disorder subgroups $(P<0.05$, Table 3$)$. 
Table 3

Comparisons of APN concentrations in 2 groups of PCOS women.

\begin{tabular}{|c|c|c|c|c|c|}
\hline & \multicolumn{2}{|c|}{ Blunted HRR Group } & \multicolumn{2}{|c|}{ Normal HRR Group } & \multirow[t]{2}{*}{$P$-value } \\
\hline & $\mathrm{n}$ & APN(ug/ml) & $\mathrm{n}$ & APN(ug/ml) & \\
\hline All patients & 23 & $10.2 \pm 3.9$ & 66 & $13.1 \pm 4.1$ & 0.015 \\
\hline Hypertension(+) & 5 & $8.3 \pm 2.6$ & 6 & $11.5 \pm 3.9$ & 0.007 \\
\hline Hypertension(-) & 18 & $10.9 \pm 4.1$ & 60 & $13.3 \pm 3.6$ & 0.023 \\
\hline Hyperlipemia(+) & 17 & $8.8 \pm 2.1$ & 46 & $11.7 \pm 3.5$ & 0.018 \\
\hline Hyperlipemia(-) & 6 & $11.9 \pm 3.0$ & 20 & $14.6 \pm 4.2$ & 0.009 \\
\hline Glucose metabolism disorder(+) & 9 & $9.0 \pm 2.7$ & 21 & $11.2 \pm 3.7$ & 0.034 \\
\hline Glucose metabolism disorder(-) & 14 & $11.1 \pm 3.4$ & 45 & $14.8 \pm 4.3$ & 0.005 \\
\hline \multicolumn{6}{|c|}{$\begin{array}{l}\text { APN, adiponectin; Hypertension (+), patients with hypertension history; Hypertension (-), patients without } \\
\text { hypertension history; Hyperlipemia }(+) \text {, patients with hyperlipemia history; Hyperlipemia }(-) \text {, patients without } \\
\text { hyperlipemia history; Glucose metabolism disorder (+), patients with glucose metabolism disorder history; } \\
\text { Glucose metabolism disorder (-), patients with glucose metabolism disorder history. }\end{array}$} \\
\hline
\end{tabular}

In multiple linear regression analysis, age $(\beta=-0.26, P<0.01)$ and hyperlipemia $(\beta=-0.13, P<0.05)$ were independently associated with the levels of APN (Table 4). The adjusted $R^{2}$ value was 0.24 for this model.

Table 4

Multiple linear regression analysis of APN concentration with clinical parameters as the dependent variable.

\begin{tabular}{|llll|}
\hline & $\boldsymbol{\beta}$ & $\mathbf{9 5 \%} \mathrm{Cl}$ & $\boldsymbol{P}$-value \\
\hline Age & -0.26 & $-0.47--0.03$ & 0.008 \\
\hline BMI & 0.21 & $0.11-0.46$ & 0.058 \\
\hline Hypertension & 0.18 & $0.05-0.39$ & 0.237 \\
\hline Hyperlipemia & -0.13 & $-0.27-0.08$ & 0.024 \\
\hline Glucose metabolism disorder & 0.41 & $0.16-0.77$ & 0.169 \\
\hline FBG & -0.05 & $-0.18-0.26$ & 0.459 \\
\hline TC & 0.39 & $0.21-0.64$ & 0.121 \\
\hline TG & -0.17 & $-0.31-0.35$ & 0.306 \\
\hline HRR & 0.34 & $0.12-0.93$ & 0.275 \\
\hline BMI, body mass index; FBG, fasting blood glucose; TC, total cholesterol; TG, triglyceride; HRR, heart rate recovery. \\
\hline
\end{tabular}

\section{Discussion}

In this study, we found that PCOS women with blunted HRR had lower values of circulating APN than those women with normal HRR. Furthermore, in multivariate analysis, age, BMI, hypertension and APN concentration were 
independent risk factors of HRR impairment.

PCOS women were characterized as elevated androgen levels, anovulation, and menstrual irregularity. Substantial evidences prove that sympathetic nervous system hyperactivity are associated with those vital symptoms [5]. Moreover, accumulating clinical data demonstrate that PCOS women have increased risks of cardiovascular diseases and metabolic disorders, compared with age-matched healthy women. Meanwhile, hypertension, exaggerated SBP response to exercise, increased musclesympathetic nerve activity, increased levels of adrenergic metabolites in the serum and urine, increased $\mathrm{HR}_{\text {rest }}$ and lower $\mathrm{HR}$ variability were reported as different manifestations of a generalized increase in sympathetic tone in PCOS women [20]. HRR, the quantification of HR decrease after exercise, is a straightforward method and a highly reproducible tool in assessingautonomic nervous function. Extensive investigations have recognized that it is not only a powerful factor predicting all-cause mortality, but also a potential marker predicting health outcomes including cardiovascular diseases [6]. It was reported that attenuated HRR was a precursor to hyperglycemia as well as an indicator of cardiovascular dysfunction [21-23]. In the past decades, HRR has been gradually evaluated in clinical researches of PCOS women. Giallauria F et al. found that HRR was lower in young PCOS women compared with healthy subjects (12.9 \pm 1.8 beats vs. $20.4 \pm 3.1$ beats) [24]. Similarly, Kaya C et al. found HRR was decreased in PCOS women compared with age- and BMI-matched women (15.4 \pm 1.9 beats vs. $24.2 \pm 3.4$ beats) [25]. Additionally, it was reported that 89 out of 243 young PCOS patients without known risk factors for cardiovascular diseases presented abnormal HRR, in which study abnormal HRR was defined as $\leq 18$ beats for standard exercise testing[26]. In the present study, our definition of blunted HRR was $\leq 12$ beats, and 23 out of 89 women exhibited blunted HRR.

Because HRR possesses such important predictive values, researchers made efforts to explore its influencing factors. Previous studies showed age was one of those factors, and our multivariate analysis result confirmed it. The prevalence of abnormal HRR was reported to be greater in older than younger individuals [13, 27-28]. This ageeffect was also supported by Buchheit et al. [29], whose study revealed HRR in children were faster compared with adolescents and adults. Physical fitness and training may also influence HRR. Several cross-sectional investigations showed that athletes or physically trained individuals had faster HRR than sedentary individuals [6, 30], so we collected the information about exercise habits of those PCOS women at enrollment. Although exercise habit wasn't associated with HRR independently, the ratio of women taking regular exercise in the Blunted HRR Group was significantly lower than the Normal HRR group.

We found increased BMI was another risk factor for blunted HRR in PCOS women. As early as in 2008, it was reported abnormal HRR was inversely correlated with BMI in overweight PCOS women [24]. Then it was found that weight loss in overweight and obese women with PCOS was associated significantly with improvements in HRR [31]. Another positive finding in the current study was hypertension was also an independent determinant of attenuated HRR in PCOS women. A recent research based in China reported that HRR was lower in hypertensive patients than non-hypertensive patients, and it was even lower in hypertensive patients with uncontrolled BP than hypertensive patients with controlled BP [32]. Moreover, a study enrolling 1,855 participants who were healthy and normotensive initially indicated that slow HRR was independently associated with the development of hypertension after average 4-year's follow-up [33]. Hypertension, as a common coexisting condition in PCOS, has complicated interactions with HRR, which was out of the present study's scope. However, it was affirmative that longitudinal HRR assessment in PCOS women was helpful both in prehypertension phase and different grades of hypertension.

Besides these above-discussed clinical parameters, some circulating factors were found to be closely correlated with HRR in PCOS, such as C-reactive protein [26, 34]white blood cells count [26] and homocysteine [34]. Elevated C- 
reactive protein and white blood cells counts are classic inflammatory markers, and high level of homocysteine is confirmed as having close involvement in endothelial dysfunction. Furthermore, adipokines, which are produced by adipose tissue, have been found to mediate the crosstalk betweenmetabolic function and autonomic nervous function in different populations during the past two decades' researches [35]. APN, the most abundant adiposereleased cytokine, has an important role in metabolism, primarily through reducing insulin resistance[36]. It not only plays an important role in whole-body energy homeostasis, but also results in protection of the vasculature, heart, lung and kidney because of its anti-apoptotic properties. In addition to those effects, it was reported that higher APN levels were associated with a more favorable development of cardiovascular autonomic function in individuals with type 2 diabetes independently of multiple confounders [10]. Another clinical observation reported that lower APN levels were related to sympathetic activation and severity of obstructive sleep apnea[37]. On the other side, Łukasz Nowak et al. found that blockade of sympathetic nervous system activity by rilmenidine increased plasma APN concentration in patients with essential hypertension[38]. Recently, it was shown that increased sympathetic activity was associated with and may modulate high-molecular-weight APN levels in premenopausal women with PCOS [13].

Although our study showed the significance difference of APN levels between Blunted HRR Group and Normal HRR Group, it was found HRR wasn't an independent factor of APN concentration after multiple linear regression analysis. However, age and hyperlipemia were correlated with APN levels independently. Previous studies have revealed that serum APN levels rise with increasing age and an elevation of $\sim 1 \mu \mathrm{g} / \mathrm{mL}$ of circulating APN for every 10 years of age, in healthy individuals [39]. Meanwhile, the significant relationship between hyperlipemia and APN was consistent with former reports. It was found serum APN was negatively related to TC, LDL-C and TG concentrations, and positively related to HDL-C concentration in nondiabetic subjects[40]. Although the mechanism that accounts for the relation between APN and serum lipids is not fully understood, it was revealed that APN was directly associated with enzymes that regulate lipid metabolism, and those associations were independent of age, sex, BMI, insulin resistance and systemic inflammation[40].

Despite those interesting findings in the current study, certain limitations should be considered. First, this was a single-center study in which only a small number of PCOS patients were available for analysis; Second, our work was cross-sectional, it would be more informative to have serial values of APN and HRR to explore causality; Third, there were some factors which were noted to influence serum APN levels, such as smoking habit [41] and moderate alcohol consumption [42]. There was no women having smoking habit or alcohol habit in our study. Current evidence about the effect of dietary fatty acids on APN values is equivocal [42], and we didn't collect the information of those women's oral daily supplements, like fish oil or conjugated linoleic acid, which might have potential effect on circulating APN values.

\section{Conclusions}

In conclusion, our study demonstrated that APN levels were significantly lower in Blunted HRR Group than Normal HRR Group. Furthermore, in multivariate analysis, age, hypertension, and APN levels were independent predictors of HRR blunt in PCOS. Further large-scale studies are needed to explore the underlying pathways of interactions between APN and autonomic nervous activities in PCOS women.

\section{Abbreviations}


PCOS: polycystic ovarian syndrome: HRR : heart rate recovery; APN: adiponectin; CI: confidence interval; BMI: body mass index; HR: heart rate; SBP: systolic blood pressure; DBP: dystolic blood pressure; FBG: fasting blood glucose; IN: insulin; AST : aspartate aminotransferase; ALT: alanine aminotransferase; TC: total cholesterol; HDL-C: highdensity lipoprotein cholesterol; LDL-C: low-density lipoprotein cholesterol; TG: triglyceride; Cr: creatinine; CPET: cardiopulmonary exercise test; BP: blood pressure; Ods: odds ratios

\section{Declarations}

\section{Acknowledgements}

Not applicable.

\section{Authors' contributions}

BL: guarantor of integrity of the entire study, study concepts, data analysis and project management; HZ and ZLL: literature research, clinical studies, data analysis and statistical analysis; WF: manuscript preparation and writing; All authors read and approved the final manuscript.

\section{Funding}

This work was partly supported by National Key R\&D Program of China (2018YFC1314700), Nature Science Foundation of Shanghai (17ZR1426400) and Wu Jieping Foundation of ClinicalPharmacist Branch of Chinese Medical Association (320.6750.19090-1).

\section{Availability of data and materials}

The datasets generated and/or analyzed during the current study are not publicly available due research design, but are available from the corresponding author on reasonable request.

\section{Ethics approval and consent to participate}

This study passed the review board of Minhang Hospital Affiliated to Fudan University Research Committee. The study followed the tenets of the Declaration of Helsinki, and informed written consent was obtained from all patients and controls after we explained the nature and possible consequences of the study.

\section{Consent for publication}

Not applicable.

\section{Competing interests}

The authors declare that they have no competing interests.

\section{References}

1. Sirmans Susan M, Pate Kristen A. Epidemiology, diagnosis, and management of polycystic ovary syndrome. Clin Epidemiol. 2013; 6:1-13.

2. Rotterdam ESHRE/ASRM-Sponsored PCOS consensus workshop group. Revised 2003 consensus on diagnostic criteria and long-term health risks related to polycystic ovary syndrome (PCOS). Hum Reprod. 2004;19:41-7. 
3. Torchen LC.Cardiometabolic Risk in PCOS: More than a reproductive disorder. Curr Diab Rep. 2017; $17(12): 137$.

4. Gui J, Wang RH.Cardiovascular autonomic dysfunction in women with polycystic ovary syndrome: a systematic review and meta-analysis.Reprod Biomed Online. 2017; 35(1):113-120.

5. Li W, Chen Y, Xu L.Association of sympathetic nervous system activity with polycystic ovarian syndrome.Clin Exp Obstet Gynecol. 2014;41(5):499-506.

6. Peçanha T, Silva-Júnior ND, Forjaz CL. Heart rate recovery: autonomic determinants, methods of assessment and association with mortality and cardiovascular diseases.Clin Physiol Funct Imaging. 2014; 34(5):327-39.

7. Obata Y, Yamada Y, Takahi Y, Baden MY, Saisho K, Tamba S, et al. Relationship between serum adiponectin levels and age in healthy subjects and patients with type 2 diabetes. Clin Endocrinol (Oxf). 2013; 79(2):204-10.

8. Christou GA, Kiortsis DN. The role of adiponectin in renal physiology and development of albuminuria. J Endocrinol. 2014;221(2):R49-61.

9. Li S, Huang X, Zhong H, Peng Q, Chen S, Xie Y, et al. Low circulating adiponectin levels in women with polycystic ovary syndrome: an updated meta-analysis. Tumour Biol. 2014;35(5):3961-73.

10. Hansen CS, Vistisen D, Jørgensen ME, Witte DR, Brunner EJ, Tabák AG, et al. Adiponectin, biomarkers of inflammation and changes in cardiac autonomic function: Whitehall II study. Cardiovasc Diabetol. 2017 ;16(1):153.

11. Barbosa-Ferreira JM, Mady C, lanni BM, lanni BM, Hotta VT, Ramires FJA, et al. Dysregulation of autonomic nervous system in Chagas' heart disease is associated with altered adipocytokines levels. PLoS One.2015; 10(7):e0131447.

12. Van De Wielle R, Michels N. Longitudinal associations of leptin and adiponectin with heart rate variability in children. Front Physiol. 2017; 8:498.

13. Shorakae S, Abell SK, Hiam DS, et al.High-molecular-weight adiponectin is inversely associated with sympathetic activity in polycystic ovary syndrome. Fertil Steril. $2018 ; 109(3): 532-9$.

14. Georgoulias P, Orfanakis A, Demakopoulos N, Xaplanteris P, Mortzos G, Vardas P, et al. Abnormal heart rate recovery immediately after treadmill testing: correlation with clinical, exercise testing, and myocardial perfusion parameters. J Nucl Cardiol. 2003; 10(5):498-505

15. Watanabe J, Thamilarasan M, Blackstone EH,Thomas JD, Lauer MS. Heart rate recovery immediately after treadmill exercise and left ventricular systolic dysfunction as predictors of mortality: the case of stress echocardiography. Circulation. 2001;104(16):1911-6.

16. Maddox TM, Ross C, Ho PM, et al.The prognostic importance of abnormal heartrate recovery and chronotropic response among exercise treadmill test patients.Am Heart J. 2008 ;156(4):736-44.

17. Cole CR, Blackstone EH, Pashkow FJ, Snader CE, Lauer MS. Heart-rate recovery immediately after exercise as a predictor of mortality. N Engl J Med.1999(18); 341: 1351-7.

18. Nissinen SI, Makikallio TH, Seppanen T, Tapanainen JM, Salo M, Tulppo MP, et al. Heart rate recovery after exercise as a predictor of mortality among survivors of acute myocardial infarction. Am J Cardiol.2003; 91(6): 711-4.

19. Nanas S, Anastasiou-Nana M, Dimopoulos S, Sakellariou D, Alexopoulos G, Kapsimalakou S, et al. Early heart rate recovery after exercise predicts mortality in patients with chronic heart failure. Int J Cardiol. 2006; 110(3): 393-400.

20. Lansdown A, Rees DA. The sympathetic nervous system in polycystic ovary syndrome: a novel therapeutic target? Clin Endocrinol (Oxf). 2012;77(6):791-801.

Page $11 / 13$ 
21. Carnethon MR, Jacobs DR Jr, Sidney S, Liu K, Study C. Influence of autonomic nervous system dysfunction on the development of type 2 diabetes: the CARDIA study. Diabetes Care. 2003(11);26:3035-41.

22. Panzer C, Lauer MS, Brieke A, Blackstone E, Hoogwerf B. Association of fasting plasma glucose with heart rate recovery in healthy adults: a population-based study. Diabetes. 2002;51(3):803-7.

23. Buchheit M, Papelier Y, Laursen PB, Ahmaidi S. Noninvasive assessment of cardiac parasympathetic function: postexercise heart rate recovery or heart rate variability? Am J Physiol Heart Circ Physiol. 2007;293(1):H8-H10.

24. Giallauria F, Palomba S, Manguso F, Vitelli A, Maresca L, Tafuri D, et al. Abnormal heart rate recovery after maximal cardiopulmonary exercisestress testing in young women with polycystic ovary syndrome. Clin Endocrinol. 2008;68(1):88-93.

25. Kaya C, Akgül E, Pabuccu R. C-reactive protein and homocysteine levels are associated with abnormal heart rate recovery in women with polycystic ovary syndrome. Fertil Steril. $2010 ; 94(1): 230-5$.

26. Giallauria F, Orio F, Lombardi G, Colao A, Vigorito C, Tafuri MG, et al..

Relationship between heart rate recovery and inflammatory markers in patients with polycystic ovary syndrome: a cross-sectional study. J Ovarian Res. 2009 ;2:3.

27. Nishime EO, Cole CR, Blackstone EH, Pashkow FJ, Lauer MS. Heart rate recovery and treadmill exercise score as predictors of mortality in patients referred for exercise ECG. JAMA. $2000 ; 284(11): 1392-8$.

28. Vivekananthan DP, Blackstone EH, Pothier CE, Lauer MS. Heart rate recovery after exercise is a predictor of mortality, independent of the angiographic severity of coronary disease. J Am Coll Cardiol. 2003 ;42(5):831-8.

29. Buchheit M, Duche P, Laursen PB, Ratel S.Postexercise heart rate recovery in children:relationship with power output, blood pH,and lactate. Appl Physiol Nutr Metab (2010);35: 142-150.

30. Trevizani GA, Benchimol-Barbosa PR, Nadal J. Effects of age and aerobic fitness on heart rate recovery in adult men. Arq Bras Cardiol. 2012; 99(3): 802-10.

31. Thomson RL, Buckley JD, Noakes M, Clifton PM, Norman RJ, Brinkworth GD. Heart rate recovery improves after weight loss in overweight and obese women with polycystic ovary syndrome. Fertil Steril. 2010;93(4): 1173-8.

32. Yu Y, Liu T, Wu J, Zhu P, Zhang M, Zheng W,et al. Heart rate recovery in hypertensive patients: relationship with blood pressure controlJ Hum Hypertens. 2017 ; 31(5): 354-60.

33. Jae SY, Bunsawat K, Fadel PJ, Fernhall B, Choi YH, Park JB, et al. Attenuated heart rate recovery after exercise testing and risk of incident hypertension in men. Am J Hypertens. 2016;29(9):1103-8.

34. Cemil Kaya , Ebru Akgül, Recai Pabuccu. C-reactive protein and homocysteine levels are associated with abnormal heart rate recovery in women with polycystic ovary syndrome. Fertil Steril. $2010 ; 94(1): 230-5$.

35. Wang ZV, Scherer PE. Adiponectin, the past two decades. J Mol Cell Biol. 2016 ;8(2):93-100.

36. George A, Konstantinos D, Christina IM, Valotassiou V, Tsikouras P, Vrachnis N, et al. The emerging roles of adiponectin in female reproductive system-associated disorders and pregnancy. Reprod Sci. 2013;20(8):87281.

37. Jamie C, Aimin X, Sidney T, Khong PI, Yao TJ, Lam D, et al. Hypoadiponectinemia is related to sympathetic activation and severity of obstructive sleep apnea. Sleep. 2008 ;31(12):1721-7.

38. Łukasz N, Marcin A, Andrzej W. Blockade of sympathetic nervous system activity by rilmenidine increases plasma adiponectin concentration in patients with essential hypertension. Am J Hypertens. 2005 ;18(11):14705. 
39. Obata Y, Yamada Y, Takahi Y, Baden MY, Saisho K, Tamba S, et al. Relationship between serum adiponectin levels and age in healthy subjects and patients with type 2 diabetes. Clin Endocrinol (Oxf). 2013;79:204-10.

40. Juan J. Díez and P. Iglesias. The role of the novel adipocyte-derived protein adiponectin in human disease: an update.Mini-reviews in medicinal chemistry. 2010; 10(9): 856-869.

41. Miyazaki T, Shimada K, Mokuno H, Daida H. Adipocyte derived plasma protein, adiponectin, is associated with smoking status in patients with coronary artery disease. Heart. 2003;89(6):663.

42. Michael M Swarbrick, Peter J Havel. Physiological, pharmacological, and nutritional regulation of circulating adiponectin concentrations in humans. Metab Syndr Relat Disord. 2008 ;6(2):87-102. 